\title{
The Relationship between Changes in Serum Glycine and Alanine Concentrations in Non-Essential Amino Acid and Milk Production in the Transition Period in Dairy Cows
}

\author{
Ken-ichi SHIBANO ${ }^{1)}$, Seiichi KAWAMURA ${ }^{2)}$, Ritsuko HAKAMADA ${ }^{2)}$ and Yozo KAWAMURA ${ }^{2)}$ \\ ${ }^{1)}$ Hata Veterinary Clinical Center, Hyogo Prefectural Federation of Agricultural Mutual Aid Associations, Kobe, Hyogo 651-1343 and \\ ${ }^{2)}$ Laboratory of Veterinary Internal Medicine, School of Veterinary Medicine and Animal Sciences, Kitasato University, Towada Aomori \\ 034-8628, Japan
}

(Received 22 April 2004/Accepted 27 September 2004)

ABSTRACT. The serum concentration of non-essential amino acid (NEAA) was measured in ten Holstein dairy cows grouped as low production $(n=5)$ and high production $(n=5)$ from one month pre-partum through four months post-partum and the relationship between production and amino acid concentrations was studied. The glycine (Gly)/NEAA ratio and the glycine/alanine ratio of the high production group were significantly higher than the low production group $(p<0.01)$. The observed decrease of the alanine (Ala)/NEAA ratio was more remarkable in the high production group than in the low production group. Measurement of Gly/Ala ratio in serum may be useful for evaluating the nutritional status of peri-parturient dairy cow.

KEY WORDS: amino-acid, dairy cow, transition period.

J. Vet. Med. Sci. 67(2): 191-193, 2005

The transition period of dairy cattle is defined as the three weeks prior to calving through the first three weeks after calving [3]. During this time the dairy cow undergoes significant physiologic changes including regeneration of the mammary gland tissue, rapid fetal growth, a reduction of dry matter intake (DMI) immediately pre-partum followed by a rapid increase post-partum, and significant repartitioning of nutrients toward lactation [1]. Serum free amino acid (FAA) concentration is influenced by muscle catabolism [1, 4], amino acid profile [6] and gastrointestinal absorption [5]. The concentration of most FAAs decreases abruptly in pre-partum cows, however, glycine (Gly) increases [5, 7, 8]. Moreover, alanine (Ala) is associated with energy metabolism through the alanine-glucose cycle between skeletal muscle and the liver. Few reports, however, have studied the relationship between serum FAA concentrations and level of milk production in dairy cattle. The purpose of this study was to investigate changes in the Gly/Ala ratios, and the concentrations of Gly/NEAA and Ala/NEAA during the period from 1 month pre-partum to 4 months post-partum in low and high producing dairy cows, while also examining the relationship between lactation performance and concentration of amino acid in serum.

The 10 cows used in this study were pregnant Holstein dairy cows that had previously calved from 2 to 7 times, and were scheduled to calve around the same time. The roughage was alfalfa, timothy, and sudan hays, to which commercial combination feed (TDN72.1\%, CP16.3\%) and mineral supplements were added in the proper amounts. The daily feed combination was TDN/DM (73.8\%), CP/DM (17.2\%), UIP/CP (35.6\%), FAT/DM (3.6\%), ADF/DM (20.7\%), NDF/DM (34.3\%), starch/DM (21\%), and DM/BW (4.1\%).

Blood collection from the jugular vein was performed at the same times of $-30,-14,1,14,30,60,90,120$ days after calving a total of 8 times. The samples were immediately cooled and separated by centrifuging for $15 \mathrm{~min}$ at $2,200 \times \mathrm{g}$ in the lab and stored at $-30^{\circ} \mathrm{C}$ until nonesterified fatty acid (NEFA) and NEAA analyses were performed.

The concentrations of Gly, Ala, serine (Ser), proline (Pro), tyrosine (Tyr), glutamine (Gln), and glutamic acid (Glu) were determined by high performance liquid chromatography. NEFA concentration was determined using a commercial kit (Wako Pure Chemicals, Osaka, Japan).

Cows in this study were grouped by milk production based on average production from the first and second official milk test results. The five cows in the low production group produced less than $45 \mathrm{~kg}(43.5 \pm 0.6 \mathrm{~kg})$ while the five cows in the high production group produced $45 \mathrm{~kg}$ or more $(50.4 \pm 4.1 \mathrm{~kg})(p<0.05)$. Groups were compared using twoway repeated measures analysis of variance (ANOVA). Unpaired Student's $t$-test was used to compare the data from the two groups.

A significant increase in serum NEFA concentration from the day after calving to one month post-partum was observed, moreover, it was significantly higher in the high production group compared with the low production group at two weeks post-partum $(p<0.01)$. Serum NEFA concentration is a reliable index of the magnitude of negative energy balance. But, no difference in NEFA concentration was observed between the two groups by ANOVA (Fig. 1).

The NEAA concentration was significantly higher two weeks post-partum compared to one month pre-partum in the high production group $(p<0.05)$, however, no difference in NEAA concentration was observed between the two groups by ANOVA (Fig. 2).

The concentrations of Gly, and Ser in serum increased from two weeks pre-partum until the early lactation period. The concentrations of Ala, Pro and Tyr in serum decreased 


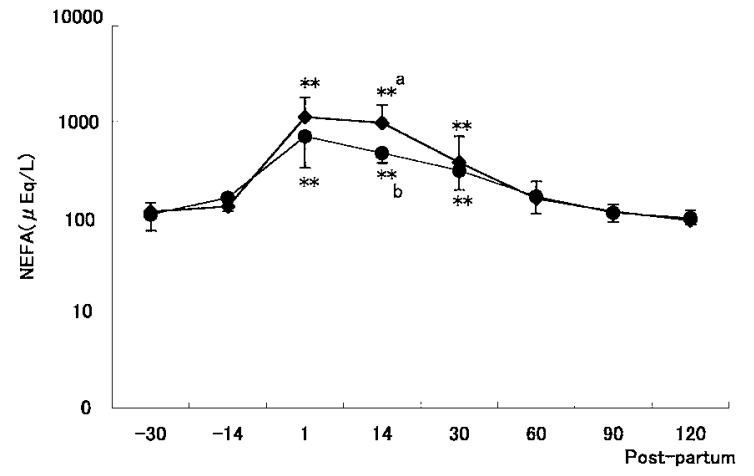

Fig. 1. NEFA concentrations in the high production group ( $\bullet)$, and low production group (). Data are shown as mean values \pm SD. Asterisks show significant differences from the value at 1 month pre-partum by Student's $t$-test $(* * \mathrm{p}<0.01)$. a-b) Values with different superscripts are significantly by Student's $t$-test $(\mathrm{p}<0.01)$.

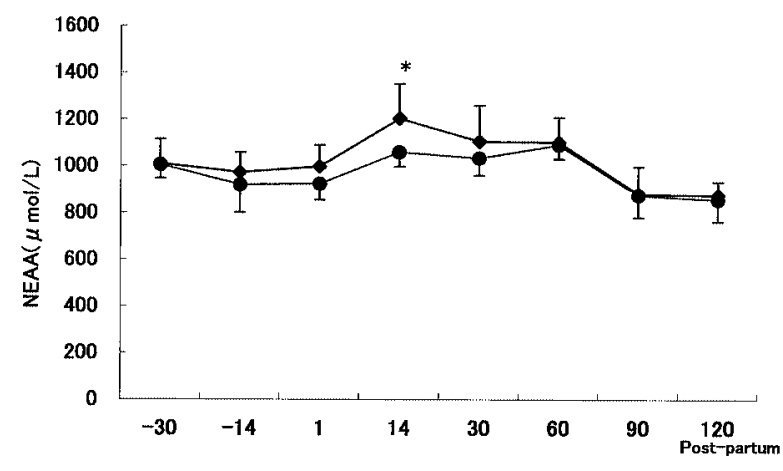

Fig. 2. NEAA concentrations in the high production group $(\diamond)$, and low production group $(\mathbf{O})$. Data are shown as mean values $\pm \mathrm{SD}$. Asterisks show significant differences from the value at 1 month before calving by Student's $t$-test (* $\mathrm{p}<0.05)$.

at calving. The concentrations of Glu, and Gln in serum decreased from two weeks pre-partum until the early lactation period.

The Gly/NEAA ratio was significantly higher in the high production group than the low production group by ANOVA $(p<0.01)$. For both groups the Gly/NEAA ratio increased from two weeks pre-partum. It increased significantly from the day after calving until two months post-partum compared to the one month pre-partum value $(p<0.01)$. Moreover, it was significantly higher in the high production group than the low production group on the day after calving and two weeks post-partum $(p<0.01)$. The observed increase is likely the result of Gly mobilization from skeletal muscle and the energy demands of early lactation in the face of depressed dry matter intake of the peri-parturient period. The Gly/NEAA ratio was significantly higher the day after calving and two weeks post-partum in the high production groups indicative of the relatively higher demands for pro-

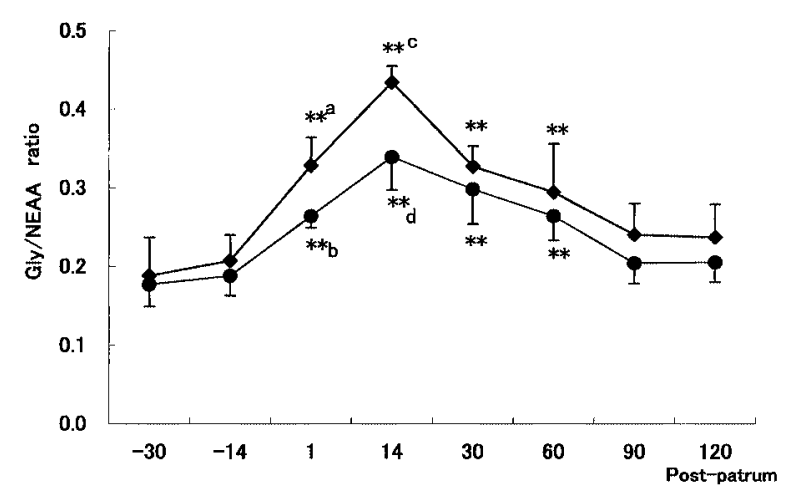

Fig. 3. Gly/NEAA ratios in the high production group $(\diamond)$, and low production group $(\mathbf{O})$. Data are shown as mean values \pm $\mathrm{SD}$. Asterisks show significant differences from the value at 1 month pre-partum by Student's $t$-test $(* * \mathrm{p}<0.01)$. A significant difference is seen between the high production group and the low production group by ANOVA $(\mathrm{p}<0.01)$. a-b), c-d) Values with different superscripts are significantly different by Student's $t$-test $(\mathrm{p}<0.01)$.

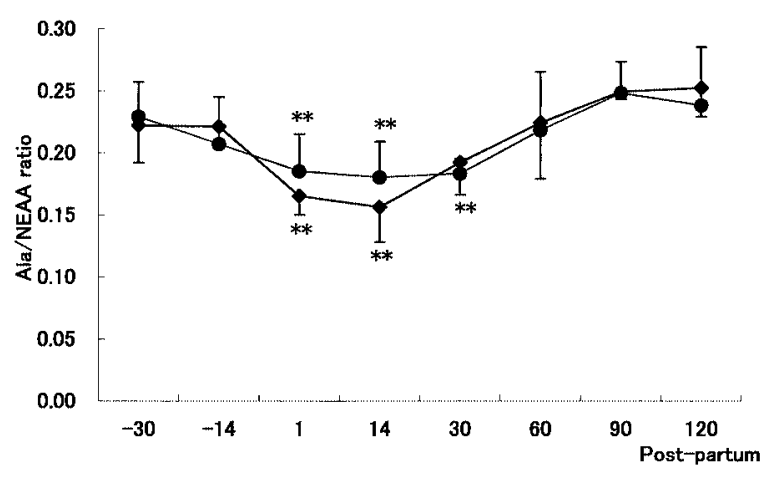

Fig. 4. Ala/NEAA ratios in the high production group $(\boldsymbol{\nabla})$, and low production group (O). Data are shown as mean values \pm SD. Asterisks show significant differences from the value at 1 month before calving by Student's $t$-test $(* * \mathrm{p}<0.01)$.

tein and energy in this group (Fig. 3).

The Ala/NEAA ratio was not different between the two groups by ANOVA. For both groups there was a tendency for it to decrease starting at two weeks pre-partum. In the low production group the Ala/NEAA ratio was significantly lower from the day after calving until one month post-partum compared to the one month pre-partum value $(p<0.01)$. In the high production group the Ala/NEAA ratio was significantly lower the day after calving and two weeks postpartum compared to the one month pre-partum value $(p<0.01)$. The change was more remarkable in the high production group than the low lactation group (Fig. 4). Since Ala is used up more rapidly than other amino acids through gluconeogenesis in the liver [2], the amount of energy required for lactation appeared to be greater prior to delivery in the high production group. The reason for the reduced 


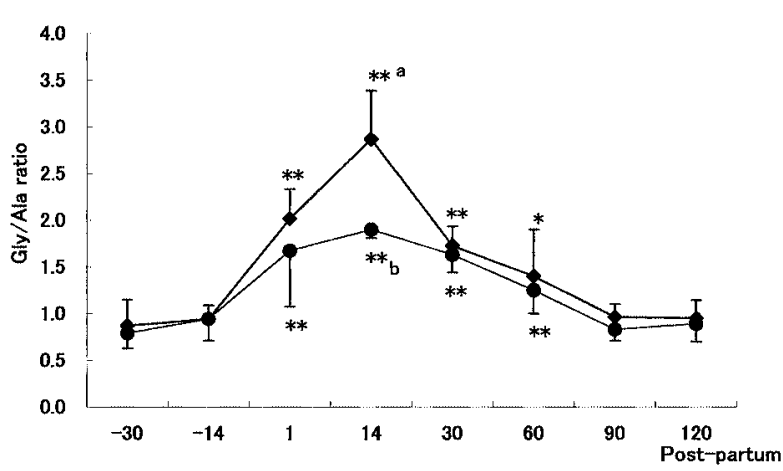

Fig. 5. Gly/Ala ratios in the high production group $(\diamond)$, and low production group (O). Data are shown as mean values \pm SD. Asterisks show significant differences from the value at 1 month before calving by Student's $t$-test $\left(* * \mathrm{p}<0.01,{ }^{*} \mathrm{p}<0.05\right)$. A significant difference was seen between the high production group and the low production group by ANOVA ( $<<0.01)$. a-b) Values with different superscripts are significantly different by Student's $t$-test $(\mathrm{p}<0.01)$.

Ala concentration in the blood is assumed to be its increased absorption by the liver for use in gluconeogenesis.

The Gly/Ala ratio was significantly higher in the high production group than the low production group by ANOVA $(p<0.01)$. For both groups the Gly/Ala ratio increased starting two weeks pre-partum. It increased significantly from the day after calving until two months postpartum compared to the one month pre-partum value $(p<0.01, p<0.05)$. Moreover, it was significantly higher in the high production group than the low production group at two weeks post-partum $(p<0.01)$ (Fig. 5). This increase can be explained by the sudden increase in demand for amino acids and energy by the udder at the onset of lactation. The response in feed intake and mobilization of body protein is thought to be responsible for the return of concentration of most amino acids in serum to their original values within a few weeks. These effects were observed beginning on the day of parturition, and on cows with fatty liver and ketosis in another our study.

In conclusion, there was an increase in energy and protein demand for lactation from before parturition to the peak lactation period. At the same time amino acid mobilization from the body tissue and gluconeogenesis were accelerated in the cows in this study. These changes were more profound in the high production animals. The major use of amino acids in cows is for synthesis of protein for maintenance, growth, pregnancy, and production of milk. As milk production of cows continues to increase, meeting the nutrient demands for this added production becomes more important. The improvement of the Gly/Ala ratio in high producing dairy cows may indicate increased milk production and milk quality.Furthermore, the Gly/Ala ratio may be a useful indicator for determining nutritional deficiency from the transition period to the peak lactation period.

\section{REFERENCES}

1. Bell, A.W. 1995. J. Anim. Sci. 75: 2804-2819.

2. Black, A.L., Anan, R.S., Bruss, M.L. and Brown, C.A. 1990. J. Nutr. 120: 700-710.

3. Grummer, R.R. 1995. J. Anim. Sci. 73: 2820-2833.

4. Korhonen, M., Vanhatalo, A. and Huhtanen, P. 2002. J. Dairy Sci. 85: 1533-1545.

5. Meijer, G.A.L., Van Der Meulen, J., Bakker, J.G.M., Van Der Koelen, C.J. and Van Vuuren, A.M. 1995. J. Dairy Sci. 78: 1131-1141.

6. Polan, C.E., Cummins, K.A. and Sniffen, C.J. 1991. J. Dairy Sci. 74: 2997-3013.

7. Shibano K., Kawamura, S. and Hakamada, R. 2002. J. Jpn. Vet. Med. Assoc. 55: 647-651.

8. Verbeke, R., Roets, E. and Peeters, G. 1972. J. Dairy Sci. 39: 355. 